\title{
Simultaneous Nanolocal Polymer and In Situ Readout Unit Placement in Mesoporous Separation Layers
}

\author{
Mathias Stanzel, Lucy Zhao, Reza Mohammadi, Raheleh Pardehkhorram, Ulrike Kunz, Nicolas Vogel, \\ and Annette Andrieu-Brunsen*
}

Cite This: Anal. Chem. 2021, 93, 5394-5402

Read Online

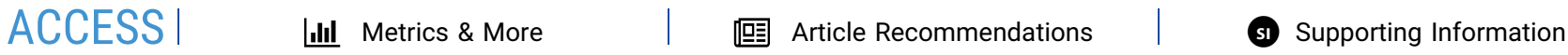

ABSTRACT: Bioinspired solid-state nanopores and nanochannels have attracted interest in the last two decades, as they are envisioned to advance future sensing, energy conversion, and separation concepts. Although much effort has been made regarding functionalization of these materials, multifunctionality and accurate positioning of functionalities with nanoscale precision still remain challenging. However, this precision is necessary to meet transport performance and complexity of natural pores in living systems, which are often based on nonequilibrium states and compartmentalization. In this work, a nanolocal functionalization and simultaneous localized sensing strategy inside a filtering mesoporous film using precisely placed plasmonic metal nanoparticles inside mesoporous films with pore accessibility control is

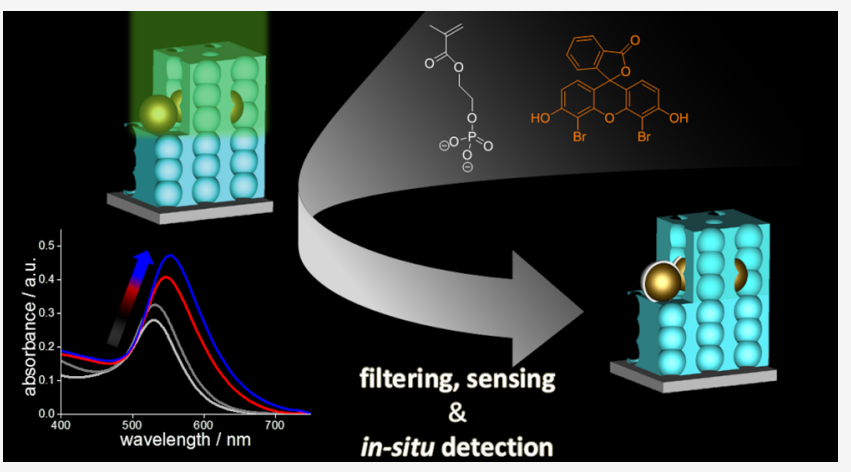
demonstrated. A single layer of gold nanoparticles is incorporated into mesoporous thin films with precise spatial control along the nanoscale layer thickness. The local surface plasmon resonance is applied to induce a photopolymerization leading to a nanoscopic polymer shell around the particles and thus nanolocal polymer placement inside the mesoporous material. As near-field modes are sensitive to the dielectric properties of their surrounding, the in situ sensing capability is demonstrated using UV-vis spectroscopy. It is demonstrated that the sensing sensitivity only slightly decreases upon functionalization. The presented nanolocal placement of responsive functional polymers into nanopores offers a simultaneous filtering and nanoscopic readout function. Such a nanoscale local control is envisioned to have a strong impact onto the development of new transport and sensor concepts, especially as the system can be developed into higher complexity using different metal nanoparticles and additional design of mesoporous film filtering properties.

\section{INTRODUCTION}

Nanoscience and nanotechnology have ever since been inspired by nature, and a variety of bioinspired materials mimicking nature have been successfully introduced. ${ }^{1-6}$ One important research goal in this context is related to compartmentalization, miniaturization, and local control of structure formation and functional placement. Therefore, a toolbox of approaches has been developed mainly using topdown and bottom-up approaches. This knowledge becomes increasingly important in the context of nanopore transport modulation to enable nature and life-inspired sustainable future water, energy, and sensing concepts. ${ }^{7-9}$ While state-ofthe-art approaches in top-down nanofabrication reach a resolution of $10 \mathrm{~nm}$ and such devices are approaching mass production, bottom-up approaches attempting to produce nanoscale devices have gained limited success. ${ }^{10,11}$ Especially polymer functionalization of pores with nanoscale resolution is still a challenge, but represents an important step toward bioinspired and dynamic transport control in technological pores. This has been demonstrated by natural channels, such as the potassium-ion channel. ${ }^{12}$ In biological channels, the arrangement of several different domains-different charges and hydrophobic side chains-leads to a dynamic, directional, and highly selective transport with high tuning rates. Interestingly, active sides of such biological ion channels are mostly concentrated to a few angstroms, which again indicates the relevance of engineering on the nanometer scale and beyond. Precise placement of responsive functionalities along nanoscale pores together with compartmentalization has to be experimentally implemented by overcoming the passive responsiveness of technological porous materials advancing toward dynamic, self-regulating, adaptive, and active materials directing flow of mass, energy, and information. ${ }^{11,13}$ These

Received: October 21, 2020

Accepted: February 24, 2021

Published: March 16, 2021 
findings have been theoretically demonstrated by Szleifer and co-workers for nanopores and by Bocquet and co-workers regarding responsive membranes. ${ }^{7,8}$

In this context, polymers and especially responsive polymers are well-established functional components that enable the precise functionalization of nanoscale pores with controlled surface coverage or charge density. ${ }^{14}$ For example, polymerfunctionalized hybrid mesoporous silica thin films show transport properties gradually tunable by controlling the polymer amount and thus controlling the functional density. ${ }^{15}$ Furthermore, applying controlled radical polymerizations allows the generation of block-co-oligomers. ${ }^{16}$ Selective functionalization of the inner or outer surface of mesoporous materials has been demonstrated by applying several approaches, for example, soft template protection, kinetic protection, or size protection as, for example, recently summarized in a review article of Tiemann and Weinberger. ${ }^{17}$ Functionalization of the outer surface of mesoporous materials allows capping of the mesopores enabling gating/switching of ionic transport ${ }^{18}$ or controlled release of guest molecules entrapped inside the mesopores, ${ }^{19}$ for instance. Such porous architectures can result in Janus-type multilayer porous materials and membranes, and several studies have been published, showing side-selective transport or oil-water separation. $^{20-23}$ Local functionalization of artificial nanochannels has been demonstrated by Jiang and co-workers using plasma-induced graft polymerization in an hourglassshaped nanochannel to generate side-specific double responsiveness. ${ }^{24}$ Applying this asymmetric modification method and cooperative $\mathrm{pH}$-responsive polyelectrolytes, namely, polyvinylpyridine and poly(acrylic acid), bioinspired artificial single-ion pumps have been realized. ${ }^{25}$ Voelcker and co-workers demonstrated multilayered surface functionalization of aluminium oxide membranes by means of multiple anodization and silanization cycles. ${ }^{26}$ Furthermore, Liu et al. demonstrated local pore wall functionalization of a single nanopore using contactless electrofunctionalization. ${ }^{27}$ Likewise, we demonstrated a strategy to introduce three functional components with precise local control into the pores of an inverse colloidal monolayer as a model system by combining orthogonal surface chemistry and control of the local wetting state of the pores very recently. ${ }^{28}$ However, these techniques allow local functionalization of small pores, but precise spatial control perpendicular to the substrate along a porous system and implementation of multiple functionalities inside nanopores still remain challenging. Pushing further local placement of polymers in small pores, nanoscale resolution would require nanoscale focusing of light in combination with photopolymerizations. This can be achieved using two photon processes, for instance. ${ }^{29}$ Alternatively, near-field modes, such as surface plasmons, offer a highly confined electromagnetic near field which is known to initiate polymerization, if compatible initiators are used. ${ }^{30-33}$ For example, successful polymer functionalization of mesoporous films deposited on planar gold or silver metal surfaces applying surface plasmons and dye-sensitized photopolymerization systems have been demonstrated in previous studies. ${ }^{34,35}$

Bachelot, Soppera and co-workers pioneered the photopolymerization induced by the high-energetic near field of silver nanoparticles, thus demonstrating nanoscale functionalization of plasmonic metal particles. ${ }^{36}$ Based on these findings, the same groups investigated not only the plasmonic optical near field of single silver nanoparticles but also the underlying mechanism of the dye-sensitized photopolymerization induced by the plasmonic nanoparticles. ${ }^{31,37}$ Furthermore, gold nanoparticles (Au-NPs) represent attractive sensing properties, as the spectral position of the localized surface plasmon resonance (LSPR) is highly sensitive to the dielectric environment. $^{38,39}$ Previous studies on composite materials using Au-NPs and inorganic mesoporous thin films demonstrated that Au-NPs can be precisely positioned with spatial control and remain accessible for chemical reactions. ${ }^{40-42}$ For instance, Angelomé et al. showed growing and branching of Au-NPs embedded in mesoporous films ${ }^{43}$ and very recently extended this concept to reaction monitoring. ${ }^{44}$

Herein, we demonstrate for the first time nanolocally limited polymer functionalization with spatial control in mesoporous silica films by taking advantage of the LSPR of Au-NPs to induce a dye-sensitized photopolymerization. Thereby, we present the preparation of a composite material consisting of mesoporous thin films and Au-NPs positioned with spatial control between the mesoporous layers and the consecutive local polymer modification. We investigate the particle loading and refractive index-based sensing sensitivity of the composite material. By that, the sensitivity of LSPR toward environmental changes in the vicinity of the particles allows in situ detection of the polymer functionalization. Moreover, the sensing sensitivity of the embedded Au-NPs only slightly decreases upon functionalization in the presented system. As the nanoparticles are synthesized before thin film deposition, the size and shape of the colloids can be easily tuned and engineered to desired properties by applying well-known synthetic protocols. Also, a variety of monomers can be applied with dye-sensitized photopolymerizations, which allows incorporation of a plethora of functionalities and thus rational design of multifunctional porous nanomaterials.

\section{EXPERIMENTAL SECTION}

Chemicals. All materials and solvents were purchased from Sigma-Aldrich, Acros Organics, and VWR and used as received unless stated otherwise. The precursor solutions for dip coating mesoporous films were prepared using the inorganic precursor tetraethoxysilane (TEOS, Acros Organics, 98\%, Geel, Belgium) in absolute ethanol (EtOH, Merck, $\geq 99.5 \%$ ), with hydrochloric acid as a catalyst $(\mathrm{HCl}, 37 \%)$ and water. Pluronic F127 (F127, BioReagent, Sigma-Aldrich, 12,600 $\mathrm{g} \mathrm{mol}^{-1}$ ) was used as structure directing agent. Microscope slides (VWR, glass, cut edges), silicon wafers (Si-Mat, Kaufering, Germany, $100 \mathrm{~mm}$ diameter, $525 \pm 25 \mu \mathrm{m}$ thickness, type P/Bor, $\langle 100\rangle$ orientation, $\mathrm{CZ}$ growth method, $2-5 \mathrm{~W}$ resistivity, polished on 1 side), and indium tin oxide (ITO, Delta Technologies, Ltd., Lovel and, CO, USA, polished float glass, $150 \times 150 \times 1.1$ $\mathrm{mm}, \mathrm{SiO} 2$-passivated/indium tin oxide-coated one surface, $\mathrm{RS}$ $=4-8 \Omega$, cut edges) were cut to the desired size using a diamond cutter, cleaned using technical grade ethanol, and dried under ambient conditions prior to dip coating of mesoporous films.

Synthesis of Au-NPs. According to a procedure from Panigrahi et al., ${ }^{45}$ a solution of $34 \mathrm{mg}$ ( $0.09 \mathrm{mmol}, 1$ equiv) of $\mathrm{HAuCl}_{4} \cdot 3 \mathrm{H}_{2} \mathrm{O}$ in $360 \mathrm{~mL}$ of water was refluxed for $10 \mathrm{~min}$. Under vigorous stirring, a solution of $45 \mathrm{mg}$ of sodium citrate ( $0.126 \mathrm{mmol}, 1.4$ equiv) in $4.5 \mathrm{~mL}$ of water (Milli-Q) was added quickly, and the solution is stirred for another $30 \mathrm{~min}$ at reflux. Then, the mixture was cooled to $0{ }^{\circ} \mathrm{C}$ and filtered $(0.4$ $\mu \mathrm{m})$. 
Synthesis of the Mesoporous Composite Material. Mesoporous silica thin films were prepared as previously described $^{46}$ via sol-gel chemistry using TEOS as an inorganic precursor and an amphiphilic triblock copolymer, Pluronic F127, as a structure-directing template that undergoes micellization upon solvent evaporation, resulting in the formation of a porous inorganic network. The molar ratio of the compounds in the precursor solution used for dip coating was 1 TEOS/0.01 F127/19 EtOH/16.4 H $\mathrm{H}_{2} \mathrm{O} / 0.015 \mathrm{HCl}$. The precursor solution was prepared at room temperature and stirred for $20 \mathrm{~min}$ before deposition on glass, silicon wafer, or indium tin oxide coated glass. The films were dip coated at a withdrawal speed of $2 \mathrm{~mm} / \mathrm{s}$ at $25{ }^{\circ} \mathrm{C}$ and $50 \%$ relative humidity. After deposition, the films were kept at $25{ }^{\circ} \mathrm{C}$ and $50 \%$ relative humidity for $1 \mathrm{~h}$. The first film is stabilized by a temperature treatment at 60 and $130{ }^{\circ} \mathrm{C}$ for $1 \mathrm{~h}$, respectively. For immobilization of Au-NPs, the mesostructured silica films were functionalized with 3-aminopropyldimethylmethoxysilane using a $0.01 \mathrm{wt} \%$ solution in anhydrous toluene. The reaction mixture and the functionalization itself have to be prepared under inert conditions. The reaction mixture was added to the mesostructured film and heated at $80{ }^{\circ} \mathrm{C}$ for $1 \mathrm{~h}$. Then, the films were rinsed with toluene and ethanol to remove unbound silane. After drying, the amine-functionalized mesostructured films were incubated in the Au-NP dispersion overnight. The $\mathrm{Au}-\mathrm{NP}$ functionalized mesostructured films were then covered with a second silica film via dip coating using the abovementioned conditions and stored at $25{ }^{\circ} \mathrm{C}$ and $50 \%$ relative humidity for $1 \mathrm{~h}$. Finally, the mesoporous composite material was obtained, after a consecutive temperature treatment at 60 and $130{ }^{\circ} \mathrm{C}$ for $1 \mathrm{~h}$, followed by a calcination step up to $350{ }^{\circ} \mathrm{C}$ with a heating rate of $1^{\circ} \mathrm{C} / \mathrm{min}$ and holding the temperature for $2 \mathrm{~h}$.

Photopolymerization. The photopolymerization in solution and in the mesoporous composite material was performed with a formulation composed of $1.22 \times 10^{-6} \mathrm{~mol}$. $\mathrm{L}^{-1}$ dibromofluorescein as a sensitizer dye, $6.71 \times 10^{-6} \mathrm{~mol}$. $\mathrm{L}^{-1} \mathrm{~N}, \mathrm{~N}$-diethylmethylamine (DEMA) as a coinitiator, and 2 $\mathrm{mmol} \cdot \mathrm{L}^{-1} 2$-(methacryloyloxy)ethyl phosphate (MEP) as a monomer dissolved in dimethylformamide (DMF). The photopolymerizable formulation was adjusted to $\mathrm{pH} 7$ with triethylamine $\left(\mathrm{Et}_{3} \mathrm{~N}\right)$ and purged with nitrogen for $20 \mathrm{~min}$. Irradiation was performed with a green LED emitting at 530 $\mathrm{nm}$ (Metrohm AG, max. 487 lumen) with a distance of $14 \mathrm{~cm}$ with respect to the reaction vessel. The light intensity in dependency of the applied current can be found in the Supporting Information (Figure S7).

Attenuated Total Reflection-Fourier Transform Infrared Spectroscopy (ATR-FTIR). Infrared spectra of the prepared mesoporous films on glass substrates were recorded using a PerkinElmer Instrument Spectrum One FT-IR spectrometer equipped with a Universal ATR Polarization Accessory (Waltham, MA, USA). All spectra were normalized to the stretching vibration of free silanol groups at $\sim 905 \mathrm{~cm}^{-1}$. The spectra were recorded using Spectrum software (Version 10.5.4.738, PerkinElmer, Inc. Waltham, MA, USA, 2016) between 4000 and $650 \mathrm{~cm}^{-1}$ with a resolution of $4 \mathrm{~cm}^{-1}$. A background and a baseline correction were automatically performed. Additional bands of the glass substrate are visible in the FTIR spectra: mesoporous film signals are partially superimposed by signals originating from the glass substrate in the region of $830-1250 \mathrm{~cm}^{-1}$ depending on the penetration depth of the ATR-IR evanescent waves. Nevertheless, due to the comparable film thickness, IR spectra of different films can be compared. All further data processing was performed in OriginPro9 (ADDITIVE Soft-und Hardware für Technik und Wissenschaft GmbH, Friedrichsdorf, Germany, 2012).

Transmission Electron Microscopy (TEM). TEM micrographs were recorded using a Philips FEI CM-20 transmission electron microscope (Philips, Amsterdam, Netherlands) equipped with an LAB-6 cathode and Olympus CCD camera and with a maximum resolution of $2.3 \AA$ operating at an accelerating voltage of $200 \mathrm{kV}$. Samples were prepared by scratching off the mesoporous films from the substrate and dispersion in filtered ethanol. After $5 \mathrm{~min}$ of sonication, scratched mesoporous films were drop-cast onto $3.05 \mathrm{~mm} \mathrm{Cu}$ grids (mesh size 200) with a Lacey carbon film (Plano GmbH, article number S166-2). The samples were dried under ambient conditions.

Scanning Electron Microscopy (SEM). SEM micrographs were obtained using a Philips XL30 FEG scanning electron microscope equipped with a tungsten cathode and a back-scattered electron yttrium aluminum garnet (BSE YAG) detector with an accelerating voltage of $15-25 \mathrm{kV}$, a $30 \mu \mathrm{m}$ aperture, and a spot size of 3-4. The samples were sputtercoated with a $7 \mathrm{~nm}$ coating of $\mathrm{Pt} / \mathrm{Pd}$. The digital micrographs were recorded over a range of magnifications at a working distance of approximately $10 \mathrm{~mm}$ using an SE2 detector.

Ultraviolet-Visible Spectroscopy (UV-vis). UV-vis spectra were recorded using an Agilent Technologies Cary 60 UV-vis spectrophotometer equipped with a full-spectrum Xenon pulse lamp single source. The spectra were backgroundcorrected using the corresponding solvent. Each spectrum was recorded between 750 and $400 \mathrm{~nm}$ at a scan speed of $600 \mathrm{~nm}$ $\mathrm{min}^{-1}$. A square aperture PMMA cell with a $1.0 \mathrm{~cm}$ path length was used for all measurements.

FEM Simulations. To calculate the absorption spectra of mesoporous composite materials, COMSOL Multiphysics software was used. A cuboid unit cell with periodic boundary conditions in the $x y$ plane and port boundary conditions in the $z$ direction was chosen. The optical constants of gold were taken from experimental data of Johnson and Christy. ${ }^{47}$ The porous matrix was simulated as an effective medium with a homogeneous refractive index calculated by the approximated volume fraction of air and the silica matrix. The refractive index of different surrounding medium was chosen according to the experimental values without dispersion for the whole spectrum range. The illumination was a monochromatic plane wave with linear polarization in the $x$ direction and traveling in the $z$ direction. Simulations were carried out between 400 and 800 $\mathrm{nm}$ in $5 \mathrm{~nm}$ steps. The simulations qualitatively reproduce all features of the experiments, including change in intensity and-most importantly-shift in resonance. Quantitative differences between experiments and simulations result from the idealized structure of the model, which assumes identical spherical particles within an identical local environment. In experiments, size distribution, deviations from spherical symmetry, surface inhomogeneities, variations in local environment, and possible deviations in the dielectric function of the synthesized particles all contribute to differences in the plasmon resonances.

\section{RESULTS AND DISCUSSION}

Composite Fabrication and Characterization. Precise polymer placement inside a nanoporous material was demonstrated by applying LSPR of Au-NPs and a dye- 
a)

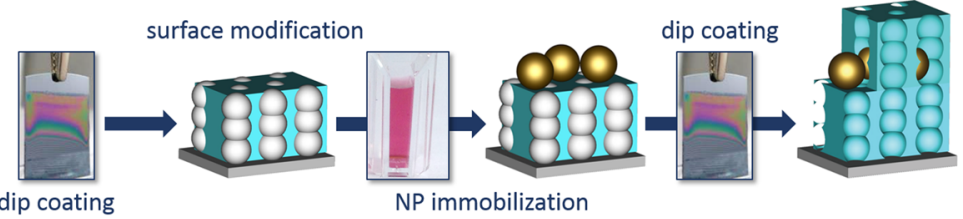

b)

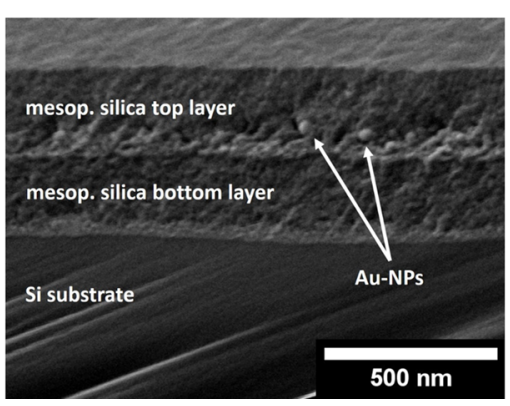

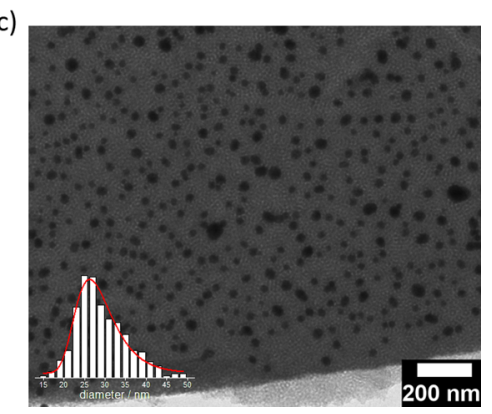

Figure 1. (a) Schematic representation of the mesoporous composite material fabrication procedure: After deposition of the first mesostructured silica thin film, Au-NPs are immobilized on the outer film surface followed by deposition of the second layer and calcination to the final porous architecture. Note that the photographs with respect to dip coating are both the same and only serve for representative purpose of the process itself showing an interference color observable on silicon wafers when the substrate had just been withdrawn from the precursor solution. (b) SEM micrograph of the composite film cross section. Scale bar: $500 \mathrm{~nm}$. (c) TEM micrograph of the composite material showing the Au-NPs with an average particle size of $26 \mathrm{~nm}$ embedded between two mesoporous silica thin films. Scale bar: $200 \mathrm{~nm}$. The Au-NP size distribution extracted from the TEM micrograph is shown as the inset.

a)

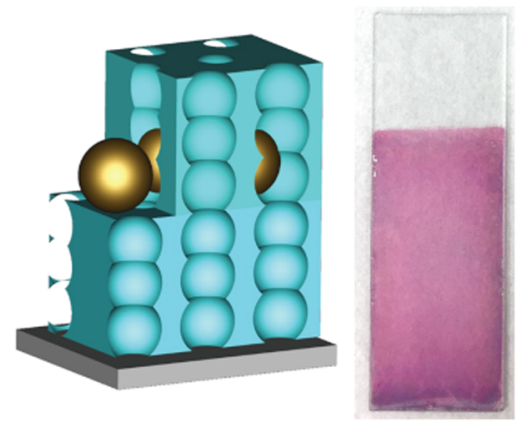

c)

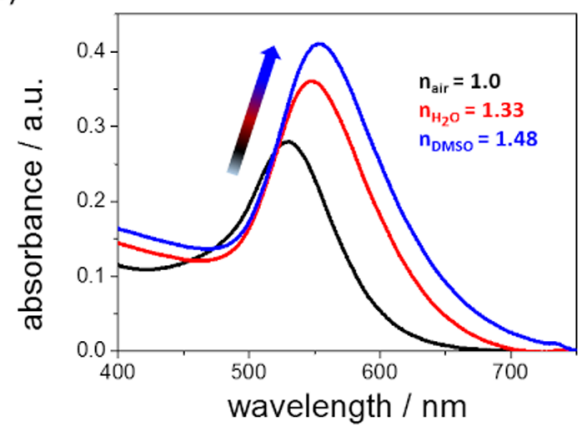

b)

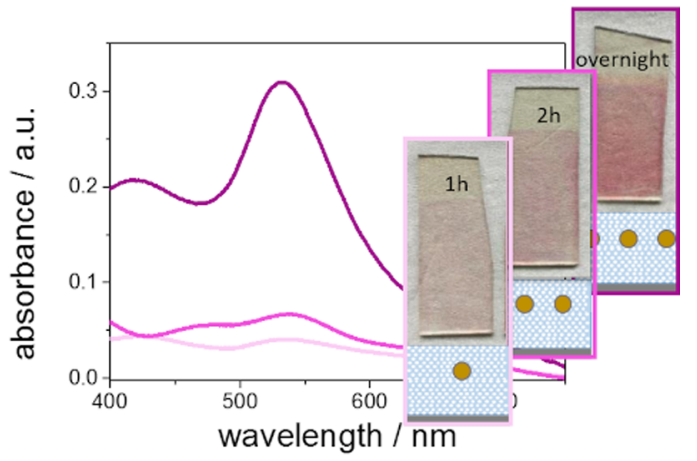

d)

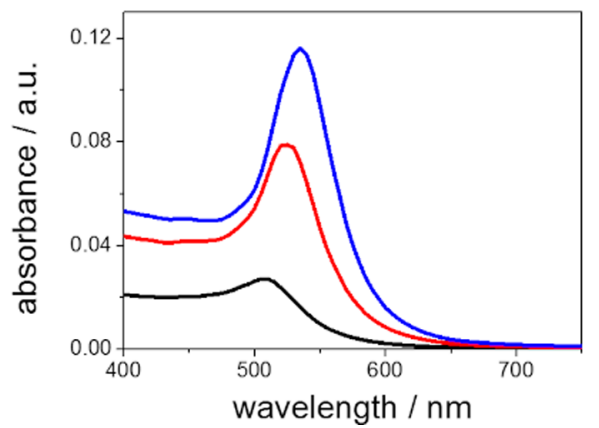

Figure 2. (a) Schematic representation of the mesoporous composite architecture and photograph of the material deposited on a glass slide. (b) Absorption spectra of composite materials in dependence of Au-NP loading. (c) Absorption spectra of the composite material in dependence of the solvents air (black curve), water (red curve), and DMSO (blue curve) obtained experimentally and (d) by FEM simulation.

sensitized photopolymerization. The Au-NP loading of the composite material and the functionalization thereof were investigated with respect to the sensing properties of the composite in experiment and simulation.

The porous composite material was composed of a mesoporous silica double layer with $\mathrm{Au}-\mathrm{NPs}$ that can be precisely positioned between the two mesoporous layers and thus along the mesoporous film cross section (Figure 1a). First, a mesostructured thin film is deposited on a substrate via dip coating using evaporation-induced self-assembly (EISA). ${ }^{48}$

For further Au-NP sensing unit deposition, amine modification of the outer silica surface allows immobilization of Au-NPs. The immobilized Au-NPs on top of the first crackfree mesostructured thin film distribute homogeneously without aggregate formation (Figures 1 and S1). To complete the mesoporous architecture, we deposit a second mesostruc- 
tured silica layer. Calcination finally resulted in the desired mesoporous composite material depicted in Figure 1. Both layers can be adjusted in film thickness allowing to precisely position the metal nanoparticle sensor and thus the nanoscale polymerization light source along the pore dimension. ${ }^{46}$

TEM and SEM micrographs (Figure 1b,c) reveal no influence of Au-NPs on the formation and structural integrity of the second mesoporous thin film, which is in accordance with similar architectures reported in the literature. ${ }^{49,50}$ Also from SEM, it can be deduced that the top layer has a smooth surface without appreciable defects. Particle size analysis of the TEM micrograph shows a narrow size distribution with a mean particle size of $26( \pm 0.5) \mathrm{nm}$. Furthermore, an average pore size of the mesoporous silica film of $11( \pm 1) \mathrm{nm}$ is obtained from TEM (Figure S13).

The Au-NP loading was investigated regarding the incubation time of the first layer in the Au-NP suspension. The coloration of the final material is visible by the eye, as depicted in Figure 2a, and characterization by UV-vis spectroscopy shows an absorption maximum at $530 \mathrm{~nm}$. Based on UV-vis spectroscopy, control of the immobilized $\mathrm{Au}-\mathrm{NP}$ amount by varying the immersing time in Au-NP solution was confirmed (Figure 2b). Here, coloration occurs due to localized surface plasmons of the Au-NPs. Taking into account a Au-NP concentration of $0.246 \mathrm{nM}$ in the aqueous suspension resulting in an absorbance of 0.85 at a wavelength of $530 \mathrm{~nm}$, a maximum Au-NP loading of $540 \mathrm{Au}-\mathrm{NP} / \mu \mathrm{m}^{2}$ on the silica surface of the first thin film can be assumed, which is in good agreement with the TEM micrograph representing an $\mathrm{Au}-\mathrm{NP}$ loading of around $300 \mathrm{Au}-\mathrm{NP} / \mu \mathrm{m}^{2}$, shown in Figure 1c. It is worth mentioning that the TEM samples were prepared by scratching off the films from their supporting substrates, thus a loss of Au-NP cannot be excluded.

Comparing the UV-vis spectrum of the Au-NP suspension (Figure S2) with the spectra observed from the mesoporous composite, it is clear that the shape of the LSPR absorption does not change upon integration into mesoporous films, indicating that the nanoparticles were stable and no aggregation occurred. This again confirms a controlled and homogenous immobilization of the particles between two mesoporous silica thin films.

The resonance conditions of surface plasmons are highly dependent on the refractive index of the Au-NP vicinity, which makes them attractive sensors in such constructions, as demonstrated in the UV-vis spectra in Figure 2c. Due to this sensitivity to the local environment, an increase in the refractive index of the surrounding medium (e.g., solvent) leads to a redshift of the LSPR absorption maximum. By varying the surrounding media of the composite material from air $(n=$ $1.00)$ to water $(n=1.33)$ or DMSO $(n=1.48)$ and thus increasing the refractive indices, the sensing sensitivity is investigated. Typically, the sensing sensitivity $S$ of an LSPR sensor is described as the ratio of the sensor output to the change in quantity measured. Here, the refractive index is considered and the sensitivity is reported in nanometers of peak shift per refractive index unit $(\mathrm{nm} / \mathrm{RIU})$

$$
S=\Delta \lambda / \Delta n
$$

Plotting the resonance wavelength against the refractive index and proceeding a linear fit allows calculation of the sensing sensitivity, which is represented by the slope of the linear fit. Although the plasmon resonance wavelength is not strictly linear with the refractive index, it is linear to a good approximation over small ranges of $n . .^{51}$ As a result of linear fitting the resonance wavelength in dependency of the refractive index obtained from experiment, a sensitivity of 51 $\mathrm{nm} / \mathrm{RIU}$ with a Pearson correlation coefficient $R^{2}$ of 0.99082 is determined for the composite material (Figure S9). Furthermore, with increasing refractive index, an increase in the absorption of the LSPR can be found, which can be attributed to the index matching of the surrounding media and silica.

The simulated spectra in dependence of the material's surrounding shown in Figure $2 \mathrm{~d}$ qualitatively agree with the experimental results. With increasing refractive index, the absorption of the LSPR shows a redshift, and a sensitivity of 55 $\mathrm{nm} / \mathrm{RIU}$ with a Pearson correlation coefficient $R^{2}$ of 0.97732 is obtained. Additionally, an increase in the absorption is also observed for the simulated spectra. Furthermore, we proved local sensing inside the porous material by covering the composite material with a hydrophobic polysiloxane thin film (Figure S10) and observing no change in the LSPR band upon change in media and thus change in refractive index (Figure S11), as the liquid did not enter the mesoporous film.

Polymer functionalization of such complex systems with nanoscale resolution is possible by combining the plasmonic features of the composite material and dye-sensitized polymerization. This should enable future multifunctional and precisely defined hierarchical nanoporous system design. Here, the absorption spectrum of the composite material overlaps well with the absorption of the dye dibromofluorescein (DBF), and a photopolymerizable formulation thereof has been investigated.

For a locally controlled polymerization induced by the LSPR of the particles, a photopolymerizable formulation containing $\mathrm{DBF}$ as sensitizing dye, $N, N$-diethylmethylamine (DEMA) as a coinitiator, and 2-(methacryloyloxy)ethyl phosphate (MEP) as monomer has been systematically studied to determine the energy threshold at which the polymerization is initiated. Such polymerization systems are very flexible, because the components can be modified independently to adjust the physical and chemical properties of the formulation with respect to viscosity, spectral sensitivity, and polymerization threshold. $^{31}$

LSPR-Induced Photopolymerization and Characterization. First of all, the polymerization has been investigated and optimized in solution to establish suitable polymerization conditions for near-field induced polymerization. To excite the sensitizing dye DBF at its absorption maximum at $525 \mathrm{~nm}$, a green LED emitting at $530 \mathrm{~nm}$ was used and the $\mathrm{pH}$ of the polymerizable solution was adjusted to $\mathrm{pH} 7$ by the addition of $\mathrm{Et}_{3} \mathrm{~N}$. The latter is crucial, since the polymerization solution becomes acidic when adding the monomer MEP, and DBF shows no absorption in an acidic environment (Figure S3). As a tertiary amine serves as a coinitiator, the influence of $\mathrm{Et}_{3} \mathrm{~N}$, which is used to adjust $\mathrm{pH}$, is investigated by polymerization without the presence of the coinitiator, and no significant influence of $\mathrm{Et}_{3} \mathrm{~N}$ was observed (Figure S4).

In order to locally limit the polymerization to the presence of the surface plasmon, the irradiation energy has to be kept below the threshold energy of the polymerization initiation. The determination of the threshold energy dosage was carried out by investigation of the photopolymerization in solution, keeping the light intensity and the irradiating distance constant. Thereby, the irradiation time is varied and the $\mathrm{C}=$ $\mathrm{C}$ monomer stretching band of MEP in the FTIR spectrum is observed. The threshold energy corresponds to a significant 
a)

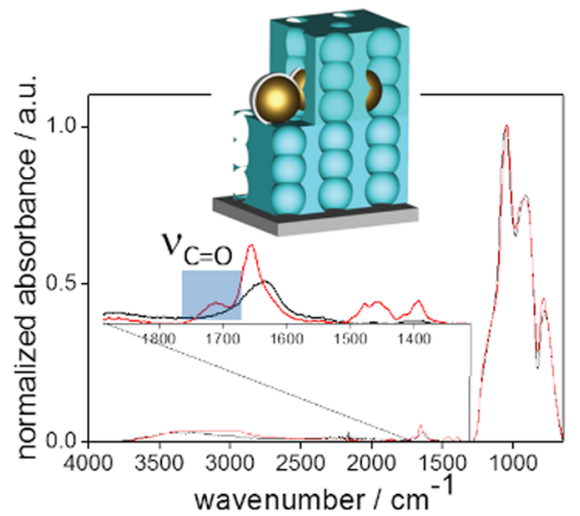

c)

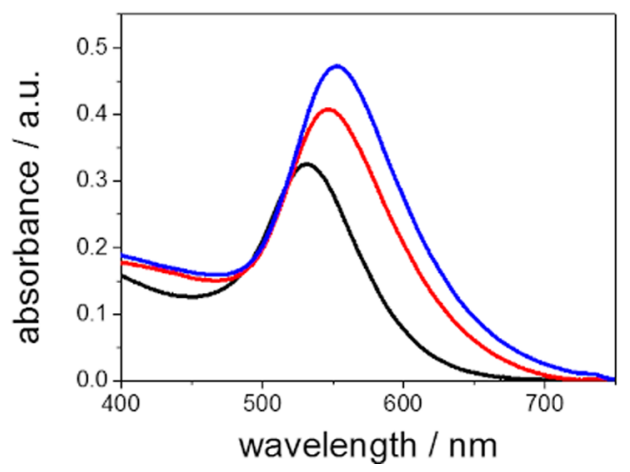

b)

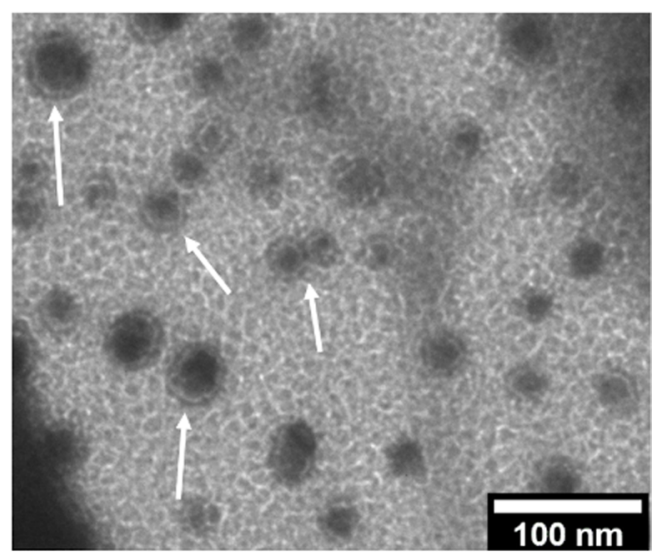

d)

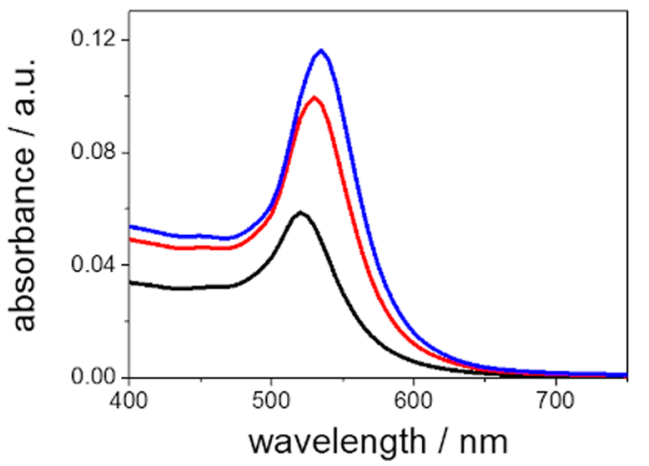

Figure 3. (a) Schematic representation of the polymer-functionalized mesoporous composite material, and FTIR spectra before (black) and after polymer functionalization (red). (b) TEM micrograph of the polymer-functionalized mesoporous composite material. Representative polymer halos of the Au-NPs are indicated by white arrows. Scale bar: $100 \mathrm{~nm}$. (c) Absorption spectra of the composite material before (black) and after polymer functionalization (red) in air and dependence of the solvents water and DMSO obtained experimentally and (d) by FEM simulation.

decrease in the absorption of the $\mathrm{C}=\mathrm{C}$ stretching band of the monomer at $1635 \mathrm{~cm}^{-1}$ in the FTIR (Figure S5) and is of significant importance to determine the energy dosage necessary for near-field polymerization. From this investigation, a threshold energy dosage of $5.7 \mathrm{~mJ} / \mathrm{cm}^{2}$ was determined under the applied conditions.

The LSPR-induced photopolymerization was thus carried out in the presence of the mesoporous composite material by applying an energy dosage of $3.8 \mathrm{~mJ} / \mathrm{cm}^{2}$, which corresponds to two-thirds of the threshold energy dosage needed to initiate the polymerization, in order to avoid free polymerization.

Characterization of functional groups using ATR-FTIR spectroscopy reveals the typical bands for mesoporous silica materials at lower wavenumbers in the range of 650 and 1280 $\mathrm{cm}^{-1}$, indicating the presence of $\mathrm{Si}-\mathrm{O}-\mathrm{Si}$ and $\mathrm{Si}-\mathrm{OH}$ bands, as discussed intensively in previous studies. ${ }^{46,52}$ After polymerization, the appearance of a carbonyl $\mathrm{C}=\mathrm{O}$ vibrational band at $1700 \mathrm{~cm}^{-1}$ can be assigned to the polymer's $\mathrm{C}=\mathrm{O}$ band (Figure 3a), which does not vanish after careful extraction overnight using DMF as a solvent, indicating covalent grafting (Figure S8). The carbonyl band is not observed, when these polymerization conditions are applied to a reference material without Au-NPs (Figure S14). Furthermore, the TEM micrograph shown in Figure $3 \mathrm{~b}$ reveals the formation of nanoscopic shells around the plasmonic Au-NPs with a thickness of approximately $5 \mathrm{~nm}$ ascribed to polymer formation after polymerization, confirming, together with ATR-IR data, the nanolocal placement of the polymer around the Au-NPs. Additionally, a three $\mathrm{nm}$ redshift of the LSPR in the UV-vis is observed after polymerization, indicating an increase in the refractive index in the vicinity of the Au-NPs (Figure S8). This demonstrates in situ detection of the AuNP's functionalization upon polymerization. Assuming total replacement of air with the polymer in the sensing range of the Au-NPs, a change in refractive index of 0.5 and a redshift of 24 $\mathrm{nm}$ were expected based on the sensitivity calculated from experimental results before functionalization. Thus, a redshift of three $\mathrm{nm}$ indicates a refractive index change of 0.059 in the sensitive vicinity of the Au-NPs, which corresponds to $12 \%$ of the maximum change expected in case of complete pore filling. Moreover, element-specific analysis using EDX spectroscopy proves the presence of phosphorus which can be attributed to the presence of PMEP (Figure S6).

Investigation of the refractive index sensitivity for additional molecules after polymer functionalization of the Au-NPs (Figure 3c,d) reveals a slight reduction of the sensing sensitivity as compared to before polymer functionalization (Figure 2) obtained from experimental and for simulated results. After polymer functionalization, a sensitivity of $44 \mathrm{~nm} /$ RIU with an $R^{2}$ of 0.99828 is observed from experimental data (Figure $3 \mathrm{c}$ and Figure S9), whereas a sensitivity of $31 \mathrm{~nm} / \mathrm{RIU}$ with an $R^{2}$ of 0.99893 is obtained from the simulated data. Due to functionalization, the sensing sensitivity dropped by $14 \%$, which is in good accordance to the calculated polymer amount of $12 \mathrm{vol} \%$ in the sensing range of the Au-NPs. However, an increase in the LSPR's absorption with higher refractive indices is also observed after polymer functionalization from both experimental and simulated spectra, which again can be attributed to the index matching of the surrounding media and silica. 
Concluding the sensitivity obtained from experiment before (51 nm/RIU) and after functionalization (44 nm/RIU), which is in very good agreement with the sensing sensitivity reported for spherical Au-NPs in dispersion solutions, ${ }^{53}$ the detection limit for aqueous protein solutions, as one sensing example, can be calculated ${ }^{54}(\mathrm{~T}=298.15 \mathrm{~K})$ as $8.6 \mathrm{wt} \%$ before and 9.5 wt $\%$ after functionalization of the Au-NPs inside the porous material, taking a signal output change of $\Delta \lambda=1 \mathrm{~nm}$ into account. Translating into values of concentration and taking bovine serum albumin as an analyte, $1.3 \mathrm{mM}$ aqueous solutions before and $1.4 \mathrm{mM}$ aqueous solutions after polymer functionalization would be detectable. Although the obtained sensitivity is lower compared to optimized commercial systems, $^{55}$ this material represents a promising starting point as an application of different colloidal metals ${ }^{56-58}$ and as well different shapes ${ }^{53,59,60}$ (Figure S14), together with ultrasensitive analytical methods, such as surface-enhanced Raman scattering (SERS), ${ }^{42,44,61}$ will improve the sensitivity significantly. In addition, the mesoporous film provides a prefilter function (Figure S12) to increase selectivity and the local placement of responsive, ligand-binding polymers enables compartmentalization, allowing new sensing concepts for future applications, as the transport properties can be easily designed upon functionalization. ${ }^{62-66}$

\section{CONCLUSIONS}

In summary, nanolocal polymer placement with precise positioning into nanoscale porous thin films and simultaneous readout of functionalization and sensing option have been demonstrated. This has been achieved by placing plasmonic gold nanoparticles as a nanolocal light source for polymerizations between two layers of mesoporous silica thin films. The position can be precisely adjusted by regulating the individual layer thickness. The nanoparticle loading can be tuned by variation of the immersion time of the chemically modified bottom layer in the aqueous nanoparticle solution. Applying dye-sensitized photopolymerization and irradiation dosage below the threshold energy, covalent polymer grafting exclusively in the nanoscopic surrounding and thus within the near-field of gold nanoparticles has been achieved. A redshift of the nanoparticle absorbance has been observed after polymerization, due to the refractive index change in the nanoparticle surrounding, which allows in situ detection of functionalization inside the nanoscale porous films. In addition, the sensing ability of the gold nanoparticles has been evaluated regarding the refractive index change, showing a sensitivity of $51 \mathrm{~nm} /$ RIU for the unmodified Au-NPs in the porous system. After nanolocal functionalization, the sensitivity decreases slightly to $44 \mathrm{~nm} / \mathrm{RIU}$, which can be attributed to the polymer shell of Au-NPs. FEM simulations of the absorption properties of the plasmonic properties are in good agreement to the experimental results obtained before and after polymer placement. This approach to functionalize nanopores and nanochannels with precise nanoscale spatial control is a key step toward the design of complex multifunctional and multiresponsive porous architectures and thus for the implementation of future water, energy management, and sensing concepts. A general classification of composite materials bearing colloidal metal and mesoporous materials into possible applications is described in detail in ref 49. The presented material design concept allows us to direct the sensing sensitivity toward highly sensitive detection playing with different colloidal metals in different shapes, together with ultrasensitive analytical methods, for example, SERS. In addition, the selectivity can be tuned using the well-known filtration, selective preconcentration, and, in general, transport and condensation control of mesopores. Furthermore, the presented material and nanoscopic sensing abilities are envisioned to open new insights into the functionalization and transport of nanopores and nanochannels themselves, providing nanoscopic local information of the confinement of nanoporous materials.

\section{ASSOCIATED CONTENT}

\section{Supporting Information}

The Supporting Information is available free of charge at https://pubs.acs.org/doi/10.1021/acs.analchem.0c04446.

Procedure of cyclic voltammetry measurements, synthesis of the hydrophobic polysiloxane thin film, synthesis of gold nanorods, surface modification of gold nanorods, immobilization of gold nanorods on the mesostructured silica surface, additional SEM image of Au-NPs immobilized on the first mesostructured layer, comparison of UV-vis spectra of aqueous $\mathrm{Au}-\mathrm{NP}$ dispersion solution and the composite material, additional data on the photopolymerization system, EDX data of polymer-functionalized Au-NPs, light intensity and photocurrent of the light source, additional data on polymer functionalization of the composite material, determination of sensing sensitivity, contact angle and data obtained from ellipsometry measurements of the hydrophobic polysiloxane thin film, UV-vis of the composite material covered with a hydrophobic layer in dependence of media, demonstration of permselectivity via cyclic voltammetry, pore size distribution of the mesoporous separation layer, reference experiment on nanolocal polymer placement, TEM of gold nanorods, and absorption spectra of gold nanorods immobilized on the first mesostructured silica film (PDF)

\section{AUTHOR INFORMATION}

\section{Corresponding Author}

Annette Andrieu-Brunsen - Ernst-Berl Institut für Technische und Makromolekulare Chemie, Technische Universität Darmstadt, 64287 Darmstadt, Germany; 이이.org/ 0000-0002-3850-3047; Email: andrieu-brunsen@ smartmem.tu-darmstadt.de

\section{Authors}

Mathias Stanzel - Ernst-Berl Institut für Technische und Makromolekulare Chemie, Technische Universität Darmstadt, 64287 Darmstadt, Germany

Lucy Zhao - Ernst-Berl Institut für Technische und Makromolekulare Chemie, Technische Universität Darmstadt, 64287 Darmstadt, Germany

Reza Mohammadi - Institute of Particle Technology, Friedrich-Alexander University Erlangen-Nürnberg, 91058 Erlangen, Germany

Raheleh Pardehkhorram - Ernst-Berl Institut für Technische und Makromolekulare Chemie, Technische Universität Darmstadt, 64287 Darmstadt, Germany

Ulrike Kunz - Department of Materials and Earth Sciences, Physical Metallurgy Group, Technische Universität Darmstadt, 64287 Darmstadt, Germany 
Nicolas Vogel - Institute of Particle Technology, FriedrichAlexander University Erlangen-Nürnberg, 91058 Erlangen,

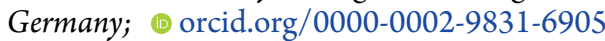

Complete contact information is available at:

https://pubs.acs.org/10.1021/acs.analchem.0c04446

\section{Notes}

The authors declare no competing financial interest.

\section{ACKNOWLEDGMENTS}

The authors thank the German Research Foundation (DFG) for financial support within the project BR4806/4 and acknowledge funding from the European Research Council (ERC) under the European Union's Horizon 2020 research and innovation programme (grant agreement no 803758). We especially thank Prof. Dr. Markus Biesalski (Chemistry Department, TU-Darmstadt) for access to interface characterization facilities and we sincerely acknowledge Dr. Meike Roskamp for fruitful discussions on plasmonic metal nanoparticle synthesis.

\section{REFERENCES}

(1) Bayda, S.; Adeel, M.; Tuccinardi, T.; Cordani, M.; Rizzolio, F. Molecules 2019, 25, 112.

(2) Benezra, M.; Penate-Medina, O.; Zanzonico, P. B.; Schaer, D.; Ow, H.; Burns, A.; DeStanchina, E.; Longo, V.; Herz, E.; Iyer, S.; Wolchok, J.; Larson, S. M.; Wiesner, U.; Bradbury, M. S. J. Clin. Invest. 2011, 121, 2768-2780.

(3) Kim, S. E.; Zhang, L.; Ma, K.; Riegman, M.; Chen, F.; Ingold, I.; Conrad, M.; Turker, M. Z.; Gao, M.; Jiang, X.; Monette, S.; Pauliah, M.; Gonen, M.; Zanzonico, P.; Quinn, T.; Wiesner, U.; Bradbury, M. S.; Overholtzer, M. Nat. Nanotechnol. 2016, 11, 977-985.

(4) Vanag, V. K.; Epstein, I. R. PNAS 2003, 100, 14635-14638.

(5) Gimenéz, C.; Climent, E.; Aznar, E.; Martínez-Máñez, R.; Sancenón, F.; Marcos, M. D.; Amoros, P.; Rurack, K. Angew. Chem., Int. Ed. Engl. 2014, 53, 12629-12633.

(6) Tonga, G. Y.; Jeong, Y.; Duncan, B.; Mizuhara, T.; Mout, R.; Das, R.; Kim, S. T.; Yeh, Y.-C.; Yan, B.; Hou, S.; Rotello, V. M. Nat. Chem. 2015, 7, 597-603.

(7) Huang, K.; Szleifer, I. J. Am. Chem. Soc. 2017, 139, 6422-6430.

(8) Marbach, S.; Kavokine, N.; Bocquet, L. J. Chem. Phys. 2020, 152, 054704.

(9) Püttmann, W.; Keil, F.; Oehlmann, J.; Schulte-Oehlmann, U. Environ. Sci. Eur. 2008, 20, 209-226.

(10) Nasrollahzadeh, M.; Sajadi, S. M.; Sajjadi, M.; Issaabadi, Z. Interface Sci. Technol. 2019, 28, 113-143.

(11) Grzybowski, B. A.; Huck, W. T. S. Nat. Nanotechnol. 2016, 11, 585-592.

(12) Doyle, D. A.; Morais Cabral, J.; Pfuetzner, R. A.; Kuo, A.; Gulbis, J. M.; Cohen, S. L.; Chait, B. T.; MacKinnon, R. Science 1998, 280, 69-77.

(13) Merindol, R.; Walther, A. Chem. Soc. Rev. 2017, 46, 55885619.

(14) Brilmayer, R.; Förster, C.; Zhao, L.; Andrieu-Brunsen, A. Curr. Opin. Biotechnol. 2020, 63, 200-209.

(15) Brunsen, A.; Calvo, A.; Williams, F. J.; Soler-Illia, G. J. A. A.; Azzaroni, O. Langmuir 2011, 27, 4328-4333.

(16) Brilmayer, R.; Hess, C.; Andrieu-Brunsen, A. Small 2019, 15, 1902710.

(17) Tiemann, M.; Weinberger, C. Adv. Mater. Interfaces 2020, 8, 2001153.

(18) Brunsen, A.; Cui, J.; Ceolín, M.; Campo, A. d.; Soler-Illia, G. J. A. A.; Azzaroni, O. Chem. Commun. 2012, 48, 1422-1424.

(19) Bernardos, A.; Aznar, E.; Marcos, M. D.; Martínez-Máñez, R.; Sancenón, F.; Soto, J.; Barat, J. M.; Amorós, P. Angew. Chem., Int. Ed. Engl. 2009, 48, 5884-5887.
(20) Lin, X.; Yang, Q.; Yan, F.; Zhang, B.; Su, B. ACS Appl. Mater. Interfaces 2016, 8, 33343-33349.

(21) Zhang, Z.; Li, P.; Kong, X.-Y.; Xie, G.; Qian, Y.; Wang, Z.; Tian, Y.; Wen, L.; Jiang, L. J. Am. Chem. Soc. 2018, 140, 1083-1090.

(22) Wang, Z.; Yang, X.; Cheng, Z.; Liu, Y.; Shao, L.; Jiang, L. Mater. Horiz. 2017, 4, 701-708.

(23) Söz, Ç. K.; Trosien, S.; Biesalski, M. ACS Mater. Lett. 2020, 2, 336-357.

(24) Hou, X.; Yang, F.; Li, L.; Song, Y.; Jiang, L.; Zhu, D. J. Am. Chem. Soc. 2010, 132, 11736-11742.

(25) Zhang, H.; Hou, X.; Zeng, L.; Yang, F.; Li, L.; Yan, D.; Tian, Y.; Jiang, L. J. Am. Chem. Soc. 2013, 135, 16102-16110.

(26) Jani, A. M. M.; Kempson, I. M.; Losic, D.; Voelcker, N. H. Angew. Chem., Int. Ed. Engl. 2010, 49, 7933-7937.

(27) Liu, J.; Pham, P.; Haguet, V.; Sauter-Starace, F.; Leroy, L.; Roget, A.; Descamps, E.; Bouchet, A.; Buhot, A.; Mailley, P.; Livache, T. Anal. Chem. 2012, 84, 3254-3261.

(28) Ochs, M.; Mohammadi, R.; Vogel, N.; Andrieu-Brunsen, A. Small 2020, 16, 1906463.

(29) Rühe, J. ACS Nano 2017, 11, 8537-8541.

(30) Nguyen, M.; Lamouri, A.; Salameh, C.; Lévi, G.; Grand, J.; Boubekeur-Lecaque, L.; Mangeney, C.; Félidj, N. Nanoscale 2016, 8, 8633-8640.

(31) Deeb, C.; Ecoffet, C.; Bachelot, R.; Plain, J.; Bouhelier, A.; Soppera, O. J. Am. Chem. Soc. 2011, 133, 10535-10542.

(32) Haggui, M.; Dridi, M.; Plain, J.; Marguet, S.; Perez, H.; Schatz, G. C.; Wiederrecht, G. P.; Gray, S. K.; Bachelot, R. ACS Nano 2012, 6, 1299-1307.

(33) Zhou, X.; Deeb, C.; Kostcheev, S.; Wiederrecht, G. P.; Adam, P.-M.; Béal, J.; Plain, J.; Gosztola, D. J.; Grand, J.; Félidj, N.; Wang, H.; Vial, A.; Bachelot, R. ACS Photonics 2014, 2, 121-129.

(34) Herzog, N.; Kind, J.; Hess, C.; Andrieu-Brunsen, A. Chem. Commun. 2015, 51, 11697-11700.

(35) John, D.; Mohammadi, R.; Vogel, N.; Andrieu-Brunsen, A. Langmuir 2020, 36, 1671-1679.

(36) El Ahrach, H. I.; Bachelot, R.; Vial, A.; Lérondel, G.; Plain, J.; Royer, P.; Soppera, O. Phys. Rev. Lett. 2007, 98, 107402.

(37) Deeb, C.; Bachelot, R.; Plain, J.; Baudrion, A.-L.; Jradi, S.; Bouhelier, A.; Soppera, O.; Jain, P. K.; Huang, L.; Ecoffet, C.; Balan, L.; Royer, P. ACS Nano 2010, 4, 4579-4586.

(38) Alvarez-Puebla, R.; Liz-Marzán, L. M.; García de Abajo, F. J. J. Phys. Chem. Lett. 2010, 1, 2428-2434.

(39) Dai, Y.; Wang, Y.; Liu, B.; Yang, Y. Small 2015, 11, 268-289.

(40) Innocenzi, P.; Malfatti, L. J. Nanoparticle Res. 2018, 20, 167.

(41) Martínez, E. D.; Boissière, C.; Grosso, D.; Sanchez, C.; Troiani, H.; Soler-Illia, G. J. A. A. J. Phys. Chem. C 2014, 118, 13137-13151.

(42) López-Puente, V.; Abalde-Cela, S.; Angelomé, P. C.; AlvarezPuebla, R. A.; Liz-Marzán, L. M. J. Phys. Chem. Lett. 2013, 4, 27152720.

(43) Angelomé, P. C.; Pastoriza-Santos, I.; Pérez-Juste, J.; Rodríguez-González, B.; Zelcer, A.; Soler-Illia, G. J. A. A.; LizMarzán, L. M. Nanoscale 2012, 4, 931-939.

(44) Zalduendo, M. M.; Oestreicher, V.; Langer, J.; Liz-Marzán, L. M.; Angelomé, P. C. Anal. Chem. 2020, 92, 13656-13660.

(45) Panigrahi, S.; Basu, S.; Praharaj, S.; Pande, S.; Jana, S.; Pal, A.; Ghosh, S. K.; Pal, T. J. Phys. Chem. C 2007, 111, 4596-4605.

(46) Herzog, N.; Brilmayer, R.; Stanzel, M.; Kalyta, A.; Spiehl, D.; Dörsam, E.; Hess, C.; Andrieu-Brunsen, A. RSC Adv. 2019, 9, 2357023578.

(47) Johnson, P. B.; Christy, R. W. Phys. Rev. B: Condens. Matter Mater. Phys. 1972, 6, 4370-4379.

(48) Brinker, C. J.; Lu, Y.; Sellinger, A.; Fan, H. Adv. Mater. 1999, 11, 579-585.

(49) Angelomé, P. C.; Liz-Marzán, L. M. J. Sol-Gel Sci. Technol. 2014, 70, 180-190.

(50) Rodríguez-Fernández, D.; Angelomé, P. C.; Soler-Illia, G. J. A. A.; Liz-Marzán, L. M. Part. Part. Syst. Char. 2017, 34, 1600428.

(51) Mayer, K. M.; Hafner, J. H. Chem. Rev. 2011, 111, 3828-3857. 
(52) Stanzel, M.; Brilmayer, R.; Langhans, M.; Meckel, T.; AndrieuBrunsen, A. Microporous Mesoporous Mater. 2019, 282, 29-37.

(53) Chen, H.; Kou, X.; Yang, Z.; Ni, W.; Wang, J. Langmuir 2008, 24, 5233-5237.

(54) Tan, C.-Y.; Huang, Y.-X. J. Chem. Eng. Data 2015, 60, 28272833.

(55) Piliarik, M.; Homola, J. Opt. Express 2009, 17, 16505-16517.

(56) Sherry, L. J.; Jin, R.; Mirkin, C. A.; Schatz, G. C.; Van Duyne, R. P. Nano Lett. 2006, 6, 2060-2065.

(57) López-Tejeira, F.; Paniagua-Domínguez, R.; Sánchez-Gil, J. A. ACS Nano 2012, 6, 8989-8996.

(58) Ma, C.; Liu, Y.; Zhao, F.; Xu, F.; Yan, J.; Li, X.; Guan, B.-O.; Yang, G.; Chen, K. J. Mater. Chem. C 2020, 8, 6350-6357.

(59) Bukasov, R.; Shumaker-Parry, J. S. Nano Lett. 2007, 7, 11131118.

(60) Michieli, N.; Balasa, I. G.; Kalinic, B.; Cesca, T.; Mattei, G. Nanoscale Adv. 2020, 2, 3304-3315.

(61) Litti, L.; Reguera, J.; García de Abajo, F. J.; Meneghetti, M.; Liz-Marzán, L. M. Nanoscale Horiz. 2019, 5, 102-108.

(62) Brilmayer, R.; Kübelbeck, S.; Khalil, A.; Brodrecht, M.; Kunz, U.; Kleebe, H. J.; Buntkowsky, G.; Baier, G.; Andrieu-Brunsen, A. Adv. Mater. Interfaces 2020, 7, 1901914.

(63) Tom, J. C.; Appel, C.; Andrieu-Brunsen, A. Soft Matter 2019, $15,8077-8083$.

(64) Silies, L.; Gonzalez Solveyra, E.; Szleifer, I.; Andrieu-Brunsen, A. Langmuir 2018, 34, 5943-5953.

(65) Andrieu-Brunsen, A.; Micoureau, S.; Tagliazucchi, M.; Szleifer, I.; Azzaroni, O.; Soler-Illia, G. J. A. A. Chem. Mater. 2015, 27, 808821.

(66) Bodelón, G.; Montes-García, V.; López-Puente, V.; Hill, E. H.; Hamon, C.; Sanz-Ortiz, M. N.; Rodal-Cedeira, S.; Costas, C.; Celiksoy, S.; Pérez-Juste, I.; Scarabelli, L.; La Porta, A.; Pérez-Juste, J.; Pastoriza-Santos, I.; Liz-Marzán, L. M. Nat. Mater. 2016, 15, 12031211. 